\title{
Implementation and Enforcement of the 3Rs Principle in the Field of Transgenic Animals used for Scientific Purposes
}

\section{Report and Recommendations of the BfR Expert Workshop, May 18-20, 2009, Berlin, Germany}

\author{
Ariane Kretlow ${ }^{1}$, Daniel Butzke ${ }^{2}$, Mario E. Götz ${ }^{1}$, Barbara Grune ${ }^{2}$, Marlies Halder ${ }^{3 *}$, \\ Frank Henkler ${ }^{1}$, Manfred Liebsch ${ }^{2}$, Rainer Nobiling ${ }^{4}$, Michael Oelgeschläger ${ }^{2}$, \\ Kurt Reifenberg ${ }^{5}$, Bernd Schäfer ${ }^{1}$, Andrea Seiler ${ }^{2}$ and Andreas Luch ${ }^{1,2}$
}

${ }^{1}$ Department Safety of Consumer Products, German Federal Institute for Risk Assessment, Berlin, Germany; ${ }^{2}$ Center for Documentation and Evaluation of Alternatives to Animal Experiments (ZEBET), German Federal Institute for Risk Assessment, Berlin, Germany; ${ }^{3}$ European Commission Joint Research Centre, Institute for Health and Consumer Protection (IHCP), In-Vitro Methods Unit, Ispra, Italy; ${ }^{4}$ Institute of Physiology and Pathophysiology, Ruprecht-Karls-University Heidelberg, Germany; ${ }^{5}$ Central Laboratory Animal Facility - CLAF, Johannes Gutenberg-University, Mainz, Germany

\begin{abstract}
Summary
In 2007, 2.7 million vertebrates were used for animal experiments and other scientific purposes in Germany alone. Since 1998 there has been an increase in the number of animals used for research purposes, which is partly attributable to the growing use of transgenic animals (Fig. 1). These animals are, for instance, used as in vivo models to mimic human diseases like diabetes, cancer or Alzheimer's disease. Here, transgenic model organisms serve as valuable tools, being instrumental in facilitating the analysis of the molecular mechanisms underlying human diseases, and might contribute to the development of novel therapeutic approaches. Due to variable and, sometimes low, efficiency (depending on the species used), however, the generation of such animals often requires a large number of embryo donors and recipients. The experts evaluated methods that could possibly be utilised to reduce, refine or even replace experiments with transgenic vertebrates in the mid-term future. Among the promising alternative model organisms available at the moment are the fruit fly Drosophila melanogaster and the roundworm Caenorhabditis elegans. Specific cell culture experiments or threedimensional (3D) tissue models also offer valuable opportunities to replace experiments with transgenic animals or reduce the number of laboratory animals required by assisting in decision-making processes. Furthermore, at the workshop an in vitro technique was presented which permits the production of complete human antibodies without using genetically modified ("humanised") animals. Up to now, genetically modified mice are widely used for this purpose.

Improved breeding protocols, enhanced efficiency of mutagenesis as well as training of laboratory personnel and animal keepers can also help to reduce the numbers of laboratory animals. Well-trained staff in particular can help to minimise the pain, suffering and discomfort of animals and, at the same time, improve the quality of data obtained from animal experiments. This, in turn, can lead to a reduction in the numbers of animals needed for each experiment.

The experts also came to the conclusion that the numbers of laboratory animals can be reduced by open access to a central database that provides detailed documentation of completed experiments involving transgenic animals. This documentation should not be restricted to experiments with substantial scientific results that warrant publication, but should also include those with "negative" outcome, which are usually not published. Capturing all kinds of results within such a database provides added value to the respective scientists and the scientific community as a whole; it could also help to stimulate collaborations and to ensure funding for future research. An important aspect to be considered in the generation of this kind of database is the quality and standardisation of the information provided on existing in vitro models and the respective opportunities for their use.

The experts felt that the greatest potential for reducing the numbers of laboratory animals in the near future realistically might not be offered by the complete replacement of transgenic animal models but by opportunities to examine specific questions to a greater degree using in vitro models, such as cell and tissue cultures including organotypic models.

The use of these models would considerably reduce the number of in vivo experiments using transgenic animals. However, the overall number of experimental animals may still be increasing or remain unaffected, e.g. when transgenic animals continue to serve as the source of primary cells and organs/tissues for in vitro experiments.
\end{abstract}

Keywords: transgenic animals, alternatives, $3 R$

\footnotetext{
* The views expressed in this article are purely those of the writers and may not in any circumstances be regarded as stating an official position of the European Commission.
} 


\section{Preface}

The number of laboratory animals used for scientific and other purposes has been steadily rising during recent years. The growing use of transgenic animals is a major contributory factor to this trend. The Federal Institute for Risk Assessment (BfR) in Berlin, Germany, invited experts from Germany and Europe to evaluate the current status and value of transgenic animal models and to identify ways to curtail their increasing use. For the purposes of this report, transgenic animals are defined as genetically modified organisms in which either genes have been selectively inactivated or xenogenous genetic material has been incorporated into their genome. Starting on May 18, 2009, 53 experts gathered at the BfR to join a three-day workshop to discuss and evaluate alternatives to the use of transgenic animals for scientific purposes and to identify important areas of future research related to this topic. Discussions were focused on opportunities and strategies to reduce the growing number of transgenic laboratory animals. Here, the so-called "3Rs principle" that seeks to replace, reduce or refine animal experiments, constituted the basis and frame of the discussions.

\section{Introduction}

There has been some inconsistency in the use of the term "transgenic" in the literature. Strictly spoken, transgenic animals are only those that carry foreign DNA within their own genome, whereas genetically modified organisms comprise transgenic animals (integration of additional genetic material by random integration after oocyte injection), knock-ins or knock-outs (certain genes being inserted or made inoperative by homologous recombination at a particular chromosomal locus in embryon- ic stem cells) and the more recently developed knock-downs (silencing of genes by insertion of DNA-constructs coding for specific small interfering RNAs or morpholino antisense oligomers). While numbers of non-transgenic animals used in animal experiments (according to the definition of "animal experiments" in Article 7 of the German Animal Welfare Act) have been decreasing consistently since 1989, the total number of animals used in experiments is increasing (Fig. 1).

However, these figures alone are far from being sufficient to understand the complexity of the issue and the impact of transgenic technology on animal welfare. In particular, numbers of animals that were killed (Article 4) or used for invasive procedures (Article 6) - both transgenics and non-transgenics - are on the rise, which is partly due to the more frequent application of primary cells in in vitro cell culture experiments. On the other hand, the use of transgenic rodents, predominantly mice, might have contributed to reduced experimentation with more highly developed vertebrates such as non-human primates. Further, if numbers of transgenic animals required to successfully address certain scientific issues were lower than they would be if one used their wildtype strain counterparts, in some areas transgenic technology might even assist in bringing down the overall levels of animals subjected to experiments.

From a scientific perspective, these novel technologies are expected to hold great promise in enabling researchers to experimentally address a myriad of complex, mostly medical problems (diseases). It thus seems likely that the pure increase in experimental approaches related to the field of transgenics may exert a much more significant impact on the total number of experimental animals in the future than any reduction or refinement could ever compensate for. Although the suffering of animals summarised in Article 4 and Article 6 might be lower when

(x1.000.000 animals)

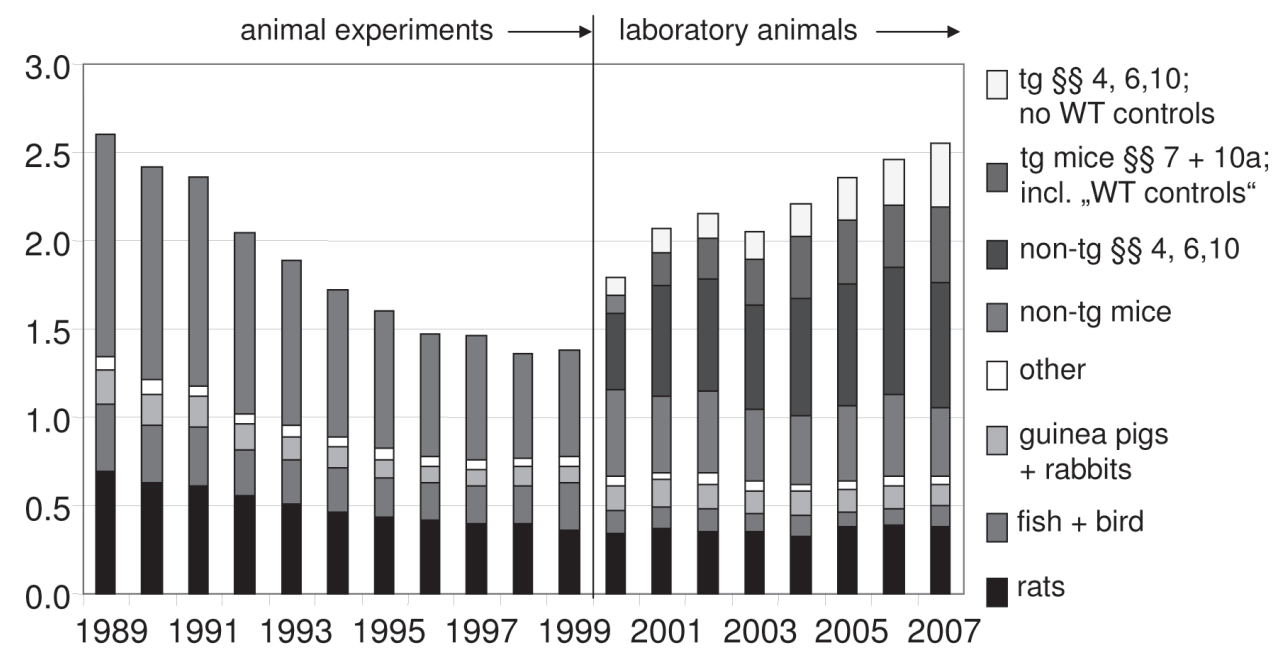

Fig. 1: Number of animals used for experiments in Germany (until 2000) and for animal experiments and other scientific purposes (since 2000).

$\operatorname{tg}=$ transgenic, $\mathrm{WT}=$ wild type. (Courtesy of Prof. Dr. Rainer Nobiling) 
compared to those actually being physically used in experiments (Article 7), the factual increase in the total number of animals used for breeding and research remains a major concern.

\section{General remarks / symposium}

A public symposium preceded the workshop. A welcoming address was given by the vice president of the BfR, Prof. Dr. Reiner Wittkowski, and by Bernhard Kühnle, Head of the Department for Food Safety at the German Federal Ministry of Food, Agriculture and Consumer Protection. This one-day event was chaired by Roman Kolar and approximately 120 stakeholders were present to discuss the use of genetically modified animals in Germany and Europe over the last few years. The first day closed with a public panel discussion hosted by Dr. Hartmut Wewetzer, Head of the Science Department of the renowned German newspaper Der Tagesspiegel. The next day, several breakout groups (BGs) were formed to discuss the following topics intensely and in appropriate depth:

BG 1: Legal background and research policies

BG 2: Gene function analysis

BG 3: Disease models

BG 4: Pharmaceutical industry

BG 5: General remarks / reduction \& refinement.

The goal of the second part of the workshop was to optimise the exchange of views from experts sharing the same expertise and to define specific recommendations on how to replace, reduce or refine the use of genetically modified organisms in the near future.

\subsection{BG 1: Legal background and research policies}

Chair: Rainer Nobiling, Institute of Physiology and Pathophysiology, University of Heidelberg, Germany

Reporter: Marlies Halder, European Commission Joint Research Centre, Institute for Health and Consumer Protection (IHCP), In-Vitro Methods Unit, Ispra, Italy

Breakout group 1 focused on the enforcement, interpretation and possible improvements to the German Animal Welfare Act, the use and usefulness of databases for already established transgenic animals (including ongoing EU projects), best practice for labelling of transgenic animals and issues concerning their patenting.

Relevant articles of the German Animal Welfare Act specifically addressing the use and production of transgenic animals are Article 7 (1), which defines experiments on animals and refers in (2) to transgenic and foster mother animals, and Article $11 \mathrm{~b}(1-3)$, which prohibits breeding of transgenic animals if the offspring is expected to suffer owing to the genetic manipulation. However, Article 11b (4) states that these restrictions do not apply to animals used for scientific purposes. Further articles are Article 4 on euthanasia of animals for scientific purposes, Article 6 (1) on the removal of organs and tissues, Article
10 on education and training and Article 10a on production of substances or microorganisms. The experts discussed the risk of double-counting transgenic animals that undergo tail biopsy for e.g. genotyping (Article 6) and subsequent experimental analysis (Article 7), and concluded that this may represent a matter of statistical reporting rather than a $3 \mathrm{R}$-related issue. The experts concluded that Article 11 on housing and breeding should be extended and include specific requirements regarding the management of the applicant's facility and training of the facility staff. The application forms, both for notification and approval, should be amended for this purpose.

The experts further recommended that characteristic features of any transgenic animal line should be extractable from a compiled list containing all relevant information such as genotype, breeding, feeding and housing, hygiene requirements and observations related to the phenotype. This information sheet should be continuously updated, adding any new observations made by others while animals are kept in experiments, thus establishing a kind of model "passport". This "passport" should be part of the files submitted as application for an animal experiment using a given transgenic animal line and should be available to other users. The experts stressed that detailed and complete phenotyping of animals should be among the criteria required to be fulfilled prior to finalisation of a particular procedure, rather than reaching an arbitrary generation number as stipulated to date.

In the context of the presentation of databases on transgenic animals, the experts underlined that any application dealing with the generation of transgenic models should demonstrate that databases such as EUCOMM, JAX, MGI or IKMC were consulted on possible existence of the model in question. Recently established "mouse clinics" (EUMODIC) are available for phenotyping mouse models using standardised protocols. The experts recommended that model developers should take advantage of this service when unable to completely characterise the phenotype of a newly generated line by their self. This would also increase the scientific value of a mouse model. In general, databases should list all phenotyping results (incl. "unfavourable") for mouse models and cell lines alike. Additionally, mouse models should imperatively be shared and submitted to cryo-archives.

For unambiguous identification purposes, the experts discussed several options. They concluded that the specific identification method(s) required for an individual animal depends on species, objectives, staff and special considerations. None of the methods (ear clipping/notching, ear tags, tattooing, transponders, etc.) should be generally excluded. However, only such methods should be applied that cause the least distress for the animal. Ear tags were less favoured because of their weight, difficulties with reading and the need for additional handling. In this respect, it is important to note that a transponder cannot always be used, for example, if NMR and/or CT techniques are part of experimentation.

Tissue from ear clipping (notches, holes) can be used for genotyping. Only in cases where more tissue might be needed should a tail biopsy be used. Other methods for genotyping based on e.g. faeces, saliva and hair are under development but not yet sufficiently robust and thus only applicable in specific cases. 
With regard to patenting of genetically modified organisms it should be noted that neither databases nor the scientific literature might reflect the actual status of the presence and availability of genetically modified organisms, since patent applications will be published no earlier than 18 months after filing (i.e. after the priority date). Applications for GMOs thus will be kept secret and information from patent databases will not be available before publication.

\subsection{BG 2: Gene function analysis}

\author{
Chair: $\quad$ Andrea Seiler, BfR, Berlin, Germany \\ Reporter: Frank Henkler, Product Safety, BfR, Berlin, \\ Germany
}

Breakout group 2 was focused on gene function analyses, which can partly be performed in cultured cells or whole animal models, for instance in zebrafish embryos, a widely used model for studying gene function in development. The experts discussed the evaluation of four alternative methods:

- transfected cell arrays

- gene function analysis in cell culture (recombination-competent $\mathrm{B}$ cell line)

- 3D stem cell / tissue models

- zebrafish (Danio rerio) embryos

Transfected cell arrays can be used as a high throughput preselection/prioritisation method and can serve as a first screening platform. Overexpression of cDNA and gene knock-down (siRNA) are both possible. Technical limitations depend on the cell type used and the availability of suitable transfection protocols. Furthermore, the time scale of the experiment is limited to $72 \mathrm{~h}$. The experts recognised that the recombination-competent B cell line represents a fairly easy and fast system to study gene function analysis by targeted gene transfer. Double or multiple knock-outs can be produced in a short time. However, the approach is currently limited to B cells. 3D tissue models offer the advantage of heterotypic cell-cell interactions and are of particular value for toxicity testing and therapeutic applications although targeted gene transfer is difficult. Finally, zebrafish embryos allow for studies without losing the complexity of an entire vertebrate organism. Due to their transparent morphology, rapid embryonic development and the availability of various different mutant strains, zebrafish currently represent the model of choice to study developmental processes. Embryos can be treated with morpholino antisense oligonucleotides to reduce the expression of specific genes and to investigate their role during normal embryonic development or in pathogenesis related to human disease. The zebrafish embryo is not a mammalian model though.

In conclusion, these alternative models have the potential to partially replace in vivo experiments where gene functions or expression patterns are to be studied. Furthermore they can aid in replacing in vivo experiments in mutagenicity studies and studies addressing protein activities and functions. They are also partially applicable as disease models. However, the cell-based in vitro models are unlikely to replace experiments in whole organisms, in particular if interactions of cell types that cannot be cultured together require consideration or when observations from the test tube demand verification in the context of entire organisms. The experts were aware of the ongoing trend of rising numbers of transgenic animals that will presumably continue in the near future, most likely as follow-ups of the various Genome Projects. They acknowledged the dedicated funding programs that should however be improved in some aspects. More funding should be directly allocated to model development. It should be acknowledged by the funding bodies that the development of alternative methods is not necessarily hypothesis-driven research and often not aimed at addressing mechanisms in the first place. Thus, the experts were aware that research on the development of alternative methods to animal experiments is currently not considered high impact research. Nevertheless, the funding agencies should allocate appropriate funding volumes despite the current lack of high impact publications. The experts also recommended that notice should be given to MEDLINE reviewers if a given publication contains novel 3R-relevant technical information (MESH-term).

To achieve a reduction of the rising number of transgenic animals the experts also agreed that the support of European efforts to standardise and connect databases (e.g. Coordination and Sustainability of International Mouse Informatics Resources- CASIMIR, InterPhenon, standards using ontologies and file formats) and the support of European and international programs for phenotyping of existing models, such as EUCOMM and NorCOMM, are highly important. In addition, retrospective reporting and archiving programs (EMMA) should become more widely known and used.

\subsection{BG 3: Disease models}

Chair: Ursula G. Sauer, Scientific Consultancy - Animal Welfare, Neubiberg, Germany

Reporter: Bernd Schäfer, Product Safety, BfR, Berlin, Germany

Recent technological advances in molecular genetics now enable large-scale production of genetically modified strains of model organisms ranging from microorganisms to mammals. The availability of these knock-out strains allows translation of basic biological phenomena into a human health perspective. In particular, mouse genetics exemplifies the translational aspect of model organism research. Overall, the use of such transgenic mammalian models significantly contributes to an increase in the number of animals used for animal experiments. Alternatively, many aspects can be pioneered and evaluated in invertebrate model systems and also in cell culture first, prior to attempting them on vertebrate models. Invertebrate models are relatively easy and inexpensive to work with and allow for precise answers for specific scientific questions. Thus their use might contribute to the reduction and replacement of animal experiments.

Four alternative models were discussed, each of them possessing particular advantages: (1) Caenorhabditis elegans is a small, free-living nematode with a transparent body consisting 
of a limited and well-defined number of cells. The unique understanding of cell lineage determination in C. elegans has already led to major discoveries in biology, including programmed cell death and RNA interference (Noble Prize 2002, 2006). During recent years this organism became the subject of an extensive integrated analysis of developmental genetics. (2) Baker's yeast, Saccharomyces cerevisiae, is a pioneer eukaryotic model. Most significantly, knowledge of the DNA sequence of the complete genome, which was completed in 1996, has altered the way molecular and cell biologists approach and carry out their studies. Some of the properties that make yeast particularly suitable for biological studies include rapid growth, dispersed cells, the ease of replica plating and mutant isolation, a well-defined genetic system and, most importantly, a highly versatile DNA transformation system. Unlike many other microorganisms, $S$. cerevisiae is viable with numerous markers. Being nonpathogenic, this yeast can be handled with few safety precautions. Large quantities of normal baker's yeast are commercially available and can provide a cheap source for biochemical studies. (3) Drosophila melanogaster has been used as a genetic model organism since the early 1900s. Genetic screens in Drosophila led to the identification of most, if not all, genes that are essential for proper embryonic development in flies as well as in vertebrates, including genes involved in various human diseases. Well established genetics provide the advantage of molecularly defined P-element insertions and deletions that span the entire genome. (4) Newly developed 3D models of skin cancer are complementary models that are instrumental in addressing issues of tumour biology including cell-cell interactions, cell proliferation and invasion.

The discussion of the various invertebrate models revealed that selection and combination of appropriate models is crucial, since one single model alone is often insufficient to explain the complexity of molecular mechanisms in pathogenesis resulting from mutations in a given candidate gene. Currently, in some cases alternative models might even not be suited to explain the diversity of symptoms. Well known examples are some mitochondrial diseases where patients often show different symptoms despite sharing the same mutation in a particular nuclear disease gene. Therefore, the different aspects of a complex system (i.e. the human body) have to be analysed using a set of model systems and also human cell lines and tissues. Among the limitations of alternative models, the understanding of the tissue-specific nature of diseases and their treatments is an important factor. Alternative model systems are not yet able to reflect the complexity of a particular disease in higher vertebrates.

Funding organisations should prioritise high quality research with clear 3Rs benefits; and an interconnection of funding institutions should be aimed for. The acceptance of results obtained from experiments in lower animals or in vitro at high impact journals is generally improvable. There are clear signs and anticipation among experts that mouse methods are usually required by editors to backup studies reporting cell culture experiments. The nomination and selection of reviewers who are comfortable in the field of alternative testing could improve this situation. Straightforward recommendations are: (i) alternative methods should be well documented and propagated in articles of well-cited journals; (ii) "alternative methods" should be indexed as such in the key word list and in literature databases; (iii) advanced networking between researchers from a particular area employing certain model organisms is necessary; (iv) sharing of data and methods between members of the academic community and industry needs to be promoted.

\subsection{BG 4: Pharmaceutical industry}

\section{Chair: $\quad$ Emily McIvor, Dr. Hadwen Trust for Humane Research, Hertfordshire, United Kingdom}

Reporter: Michael Oelgeschläger, ZEBET, BfR, Berlin, Germany

In the pharmaceutical industry, relatively low numbers of transgenic animals are used as disease models, for targeting pharmacological compounds and for antibody production, infection and vaccine studies. Some transgenic animal models allow the specific and highly sensitive detection of biological responses by in vivo bio-imaging technologies. These non-invasive monitoring systems allow the accumulation of more data with fewer animals and the detection of biological responses at lower drug concentrations and, thus, might help to refine or even reduce animal experiments.

Sharing of transgenic animals is in some cases hampered by patents, which can result in high purchase costs, and the poor characterisation of most animal strains. A depository or a global easy-to-use database, similar to that initiated by the Eumorphia project, providing standardised information about the animal models already available seems necessary. Furthermore, encouraging the publication of non-technical abstracts or results obtained from transgenic lines that appear phenotypically normal may help prevent duplication and would provide added value to the animal model as well as the research that generated them.

The use of antibodies generated without animals, e.g. by phage display, should be supported by the institutions providing research grants, since these antibodies may be more expensive. Again, the development of a depository or database of available antibodies, similar to the Developmental Studies Hybridoma Bank at the University of Iowa, including detailed technical information for research purposes is also desirable.

In preclinical experimentation, cellular systems for the prediction of toxic side effects are faster and cheaper compared to in vivo systems and allow the analysis of safety aspects, such as the benefit/risk ratio, early on. The experts noted that the provision of data from retrospective assessments of clinical studies could improve the quality of future preclinical studies.

In vitro experiments based on well characterised, stable immortalised cell lines that allow reliable and reproducible analysis of pharmacological effects have the potential to replace some animal experiments. In particular, the development of functional assays, including reporter gene assays and in vitro systems that allow the characterisation of complex immune responses, like the MIMIC system from VAX-Design, are promising approaches. There is, however, a need for continuous and increased financial support for the development, further characterisation and validation of these methods. 
The communication between academia and the competent authorities and the harmonisation of data requirements need to be improved in order to expedite regulatory acceptance (e.g. ICH, EMEA, FDA). The same holds true for the communication between different scientific disciplines, in particular with and between upcoming technologies, including QSAR, bioinformatics, omics and systems biology.

\subsection{BG 5: General aspects / reduction \& refinement}

\author{
Chair: $\quad$ Kurt Reifenberg, Central Laboratory Animal \\ Facility - CLAF, Johannes Gutenberg-University, \\ Mainz, Germany
}

Reporter: Manfred Liebsch, ZEBET, BfR, Berlin, Germany

Breakout group 5 focused on general aspects regarding reduction and refinement strategies. One major issue addressed in this group was the topic of tail biopsies. The experts agreed that taking tail tip biopsies is a routine procedure for diagnostic purposes (genotyping) and should not be considered a genuine animal experiment. This view is supported by scientific results showing that the distress (heart rate, body temperature, motility) of mice exposed to tail tip biopsies was comparable to that of animals that were only restrained. Tail tip biopsies are only allowed to be taken once. Routine genotyping of juvenile mice using hair follicle samples is not efficient and reliable, but still considered suitable as a tool to re-genotype adult mice (e.g. C57BL/6 >50 days). Research on pain and stress assessment as well as determination of appropriate humane endpoints should be supported. Early recognition of stress and pain situations should be part of pain management; simple and reliable instruments for pain and stress detection need to be established.

\section{Recommendations}

\section{Generation of transgenic lines}

1. New transgenic animals should be generated and bred under best practice conditions and animal welfare standards in compliance with existing guidelines such as those of FELASA/GV-SOLAS (http://www.felasa.eu/docs/WG_ transgenics_20070330.pdf or http://www.gv-solas.de/auss/ gen/index.html)

2. Phenotyping must follow minimum requirements for cataloguing, which allows addition of data and information as it arises. Thus, documentation sheets should be modified:

- continuous update (e.g. phenotype) of documentation sheet should be made possible

- should be presented with the application for an experiment on animals

- should be handed over with the model to new users as a kind of "passport".

3. Well-defined pure genetic backgrounds, e.g. C57BL/6, are recommended as the starting strains for transgenesis.

4. Mouse models should be shared and submitted to cryo-ar- chives. These archives will then provide gametes and embryos of the desired line to requesting scientists.

5. All phenotyping results (including negative data) should be listed in the databases of live animals, gametes, embryos and cell lines derived thereof.

6. The scientific value of mouse models can be increased by standardised characterisation in mouse clinics. However, this requires internationally agreed characterisation SOPs to be enforced by regulatory bodies and funding agencies.

7. Material transfer agreements should be obligatory. Existing barriers to material transfer, such as research competition, funding or defeating patent applications, thus need to be addressed.

8. Support of European efforts to standardise and connect databases (CASIMIR, Coordination and Sustainability of International Mouse Informatics Resources; InterPhenon, standards using ontologies and file formats) should be improved.

9. Support of European and international programmes (EUCOMM, NorCOMM) for phenotyping existing models should be improved.

10. Retrospective reporting (research and publication records of applicants who seek funding for animal experiments) should be considered by funding and regulatory authorities. The experts encourage that authorities demand feedback on previous projects. Funding agencies must be encouraged to consider the value of publishing negative findings and to agree that this may not impede future funding opportunities for the individual researcher.

11. Archiving (EMMA) programmes should become more widely known and used.

12. Transgenic animals should be generated by the most competent staff. Newcomers to the field should gain experience in collaborations or use commercial services, SOP by EUMORPHIA/EMPReSS.

\section{Application form for animal experimentation}

1. Application files should demonstrate that databases were consulted, e.g. EUCOMM, JAX, MGI, IKMC.

2. Specification of application forms should be improved, e.g. at present Article 11 of the German Animal Welfare Act does not require the provision of information on whether transgenic animals are kept and bred.

3. Article 11 - authorisation for housing and breeding should be extended to specific requirements of management, e.g. staff (specific education, training, observation skills).

4. Ethical review/biostatistics should be improved. However, unreasonable delays should be avoided.

\section{Identification of animals}

1. In general, the method that causes the least distress to the animal should be used for identification purposes. This will depend on the species and objectives. While ear punching is critically discussed, toe clipping is generally discouraged. For temporary identification, the use of permanent marker pens should be considered; tattooing and transponders could however be used for long-term studies. 


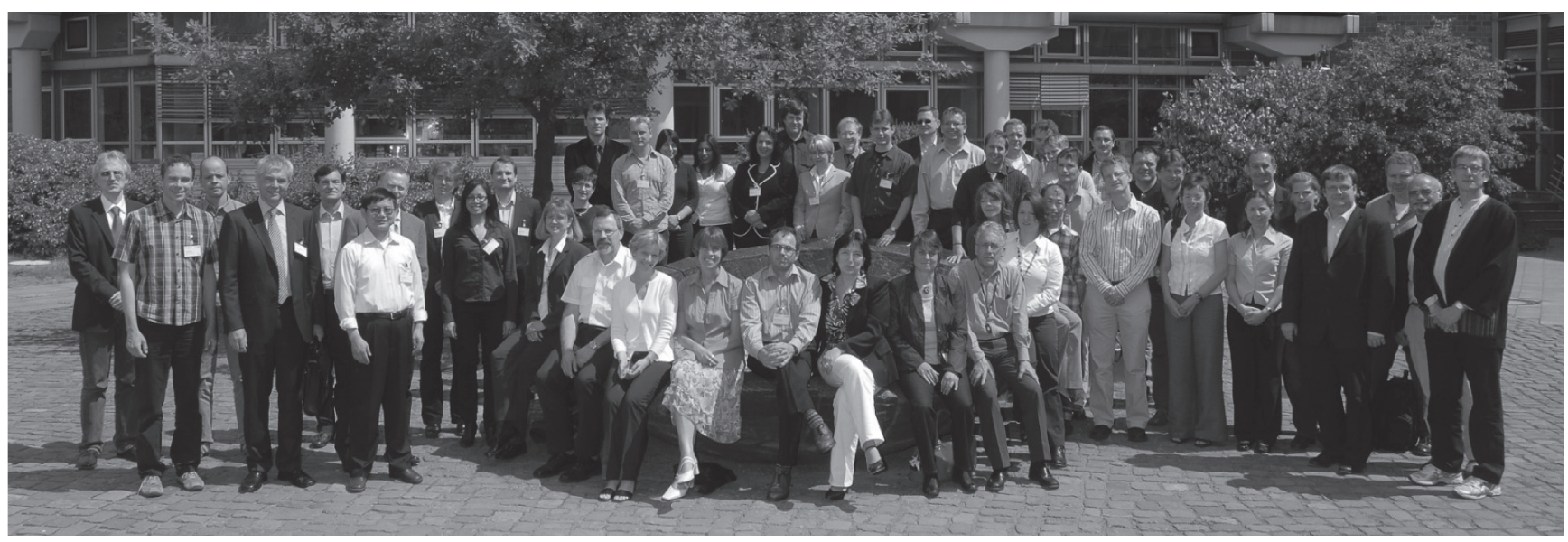

Workshop participants in front of the main building

2. For the identification of mice, ear tags are less favourable since small numbers are difficult to read, requiring additional handling of the animal and thus causing additional distress. Also, these tags may tear down ears owing to their weight.

\section{Publications}

1. Indication that publications containing novel 3R-relevant technical information should be provided to bibliographic databases, e.g. MEDLINE (MESH-term).

\section{Genotyping of animals/Tail biopsies}

1. In general, the genotyping method that causes the least distress to the animal should be used. Non-invasive methods such as analysis of faeces, saliva and hair should be used whenever possible. If chimeric mice are to be genotyped, two different sources of DNA must be considered.

2. If tissue has been gained from the identification method, this should be used for genotyping. Thus, protocols to retain integrity and for storage procedures are needed.

3 . Tail biopsy: risk/problem of counting the animals twice according to Art 6(1), 7(1)

- Not a 3Rs problem; relates to statistics and not to the goal of the workshop implementing the 3Rs.

- Proposal to carry out an inquiry on the classification of tail biopsied animals for either breeding or genotyping purposes up to generation F2, which is mandatory when generating a transgenic line. Major research institutions should be addressed, e.g. DKFZ (German Cancer Research Center), Heidelberg University, Hannover Medical School (MHH), MDC Berlin and Munich.

\section{Concluding Remarks}

The experts agreed that the strategy with the greatest potential to reduce the number of laboratory animals in the near future is unlikely to be the complete replacement of transgenic animal models, but rather addressing open scientific questions to a greater degree using in vitro models such as cell and tissue cultures. The use of such models would reduce animal numbers also in the area of experiments that could not yet be replaced and would contribute to alleviate animal suffering ("reduction" and "refinement"). Furthermore, state of the art education and training of staff is crucial to minimising stress and discomfort of laboratory animals, thereby contributing to improved reliability and to reduced variability of acquired experimental data. Regarding inevitable genotyping of animals, tail biopsies should not be considered as animal experiments according to Article 7 of the German Animal Welfare Act. The experts recommended the establishment of one central database listing all available transgenic lines that would allow users to purchase cryo-preserved material at a reasonable price. The experts further emphasised that public funding of the development and validation of new alternative methods must be significantly improved. Only through intensified efforts across all scientific disciplines and regulatory requirements might it become possible in the medium term future to meet the ambitious expectations of politicians, animal welfare groups and the general public to reverse the current trend and to sustainably reduce the overall numbers of animals, including transgenics, used for scientific experiments and other purposes.

\section{Acknowledgements}

The authors express their special gratitude to Drs. Ursula G. Sauer and Emily McIvor and would like to thank everybody who participated in the workshop and contributed to its success.

\section{Corrrespondence to}

Dr. Ariane Kretlow

BfR - Federal Institute for Risk Assessment

Thielallee 88-92

D-14195 Berlin

e-mail: Ariane.Kretlow@bfr.bund.de 


\title{
Annex:
}

\section{Abstracts presented at the BfR expert workshop on "Implementation and enforcement of the 3Rs principle in the field of transgenic animals used for scientific purposes"}

\section{The use of transgenic animals for scientific purposes: aims, numbers, trends}

\author{
Rainer Nobiling \\ Institute of Physiology and Pathophysiology, University of Heidelberg, Germany
}

The increasing use of genetically modified animals ("transgenic" animals) in science and technology is suspected to be the main cause for the rising numbers of experimental animals. However, a detailed comparison demonstrates that the enhanced use of these animals represents less than $50 \%$ of the increase of the total number of animals used for scientific and other purposes.

The trend in Germany since 2000 is much better described by the increasing use of animals for scientific purposes other than animal experiments, i.e. immunization, cell culture, isolated organs, and other alternatives, irrespective of whether the donor animals are "transgenic" or laboratory animals without any genetic manipulation. Although in this field the use of transgenic animals is increasing, this portion represents less than $50 \%$. On the other hand, for animal experiments, according to the definition in the German animal welfare act, the increasing number of transgenic animals reached a fraction of about $11 \%$. Here, the number of non transgenic animals is decreasing to a comparable extent. As a consequence, the total number of animals used in experiments is increasing slowly, or has remained nearly constant in the last few years, whereas that for alternative purposes continuously rises and will reach the one for animal experiments in the animal statistics for 2008 or 2009.

Transgenic animals in general enable the transition to more refined experimental models, in particular by the enhanced use of conditional and/or organ specific genetic modifications such as gene knockout. Moreover, improved generation and breeding methods will enable research with better standardization, and therefore, the increased number of animals in experiments may represent only a transient state.

In principle the German trends do not differ much from the European development, where after a decrease until the year 2000 , now a slight increase is to be seen, with a present level of 12 million animals in the EU 25. This number means that about one animal per an individual human lifetime is used in experiments and another one for alternative research. Transgenic animals have the potency to decrease the first of these numbers, whereas the use of alternatives will continue to increase for a certain number of years despite the enhanced use of transgenic models. Here, the promotion of biomedical research will be reflected.

\section{The use of transgenic animals from an animal welfare point of view}

\author{
Ursula G. Sauer \\ Scientific Consultancy - Animal Welfare, Neubiberg, Germany
}

In accordance with the German Animal Welfare Act, animal experiments are only permissible if the foreseen pain, suffering and distress are considered to be ethically acceptable in relation to the goal of the experiment. Severely distressful animal experiments are only permitted if they are of outstanding importance for substantial needs of humans or animals, including the solving of scientific problems. Accordingly, I will start out my presentation by discussing the distress of genetically modified (GM) animals. This includes the distress animals endure during the genetic engineering, the distress due to the breeding of GM animal lines as well as the distress due to the housing conditions of GM animals, and, finally, the distress due to the pro- 
cedures they are submitted to. I will give an overview on those research areas, in which GM animals are used and discuss the scientific significance of such experiments. In this context, I will refer to means to establish the benefit of an experiment. Based upon these considerations of the distress of the animals and the outcome of the experiments, I will weigh up whether animal ex- periments with GM animals are to be considered permissible in accordance with the German Animal Welfare Act. I will briefly list non-animal test methods in gene technology and conclude by discussing special ethical problems, which arise from the point of view of animal welfare organisations due to the genetic modification of animals.

\section{Application of the 3Rs Principle in research involving transgenic animals}

\author{
Reinhart Kluge \\ DifE, Potsdam-Rehbrücke, Germany
}

The implication of humane principles in biomedical research with animals is a long lasting topic including many different points of view and a lot of interactions among the individual ideas. Interestingly, the original paper of Russell and Burch (1959) shows the experiment to be in the focus of the 3Rs concept.

After the second world war a variety of new fields of research emerged including biomedical questions which appeared to be more and more investigated applying laboratory animals. Consequently, not only the number of laboratory animals increased very rapidly but also the demand on the hygienic and later on the genetic quality of the models.

In the following years, the establishment of Societies of Laboratory Animal Science resulted from those working groups performing biomedical research including the application of animals. The objectives of the SOLAS working groups primarily concern two of the three Rs: reduction and especially refinement.

It is about twenty years that genetically modified animals are included in research work, involving

targeted mutations of endogenous genes as well as the addition of foreign genes.

In many aspects transgenic animal models appear to be similar to conventional ones. However, during establishment there is a period of "in vitro" work which can influence the quality and the time of the subsequent sections of establishment and thus contributes to reduction and refinement (e.g. origin and genetic quality of embryonic stem cells).

After establishment the following intervals can be defined for genetically modified animals:

- breeding and multiplication (production of target genotypes)

- experiment: application of the target phenotypes in biomedical studies

Comparable to conventional models up to date methods, expert knowledge and continuous training are required for establishment and application of transgenic models. This is especially important for the following scopes:

- genetics on the molecular level including biochemical means (stem cells, gene constructs, conditional gene targeting, etc.)

- population genetics and classical breeding (breeding methods, genetic control, etc.)

- animal care and maintaining (handling, behaviour monitoring, documentation, etc.)

- experiment (handling, specific physiology, behaviour monitoring, anaesthesia, etc.)

- experimental analysis (statistical means, etc.)

Considering all these points a lot can be achieved concerning at least reduction and refinement and to meet simultaneously the experimental targets.

\section{Databases for genetically modified organisms / The EUCOMM project}

\section{Roland Friedel \\ Helmholtz Zentrum München, Munich, Germany}

Functional analysis of the mammalian genome is an enormous challenge for biomedical scientists. To facilitate this endeavor, the European Conditional Mouse Mutagenesis Program (EUCOMM) aims at generating up to 13,000 mutations by conditional gene trapping and conditional gene targeting in mouse embryonic stem (ES) cells. The leading partners of the EUCOMM consortium are the Helmholtz Zentrum München, Germany and the Wellcome Trust Sanger Institute, UK (for a complete list of all 9 partners see www.eucomm.org). The EUCOMM project closely interacts with two other international consortia, the U.S. KOMP project and NorCOMM in Canada, in the framework of the International Knockout Mouse Consortium (IKMC). It is the common goal of these partners to generate conditional mutations in mouse ES cells for the entire mouse genome. The EUCOMM 
key goals are mutating up to 5,000 genes by gene trapping and up to 8,000 genes by gene targeting in mouse embryonic stem (ES) cells. The mutations can be rendered conditional, so that each mutation can be activated by Cre recombinase in a timeand tissue-specific manner. EUCOMM distributes its resource to the scientific community (see http://www.eucomm.org and http://www.eummcr.org).

The main databases used in the field of mouse genetics are sequence based genome browsers (UCSC, Ensemb1, NCBI), gene based databases that include also data on published mutants (MGI), and databases of resource centers that distribute mouse ES cells and mouse strains (JAX, EMMA, KOMP, EUCOMM). The upcoming website of the International Knockout Mouse Consortium (IKMC) will provide a central portal that links all of the above databases. The open access to databases that publish all data about phenotypes, available ES cells and mice will greatly reduce double efforts in the generation of transgenic mice.

\title{
Patent law and genetically modified organisms
}

\section{Berthold Bettenhausen \\ Patent attorney, Munich, Germany}

The patent law is directed to inventions in general regardless of the technical field. However, in the last years several specific regulations concerning biological inventions have been implemented into the law which are discussed.

- Short introduction into patentable inventions

- Exceptions to patentability

- How to deal with biological inventions

- Are living organisms, e.g. GMOs patentable?

- Patentability of animals after implementation of Directive 98/44/EC into German patent law

- When is the use of a patented animal a patent infringement?
In view of the previous talks it should be considered that

- Patent applications will be published only 18 months after filing (i.e. after the priority date), meaning that applications for GMOs will be kept secret and information from patent databases will not be available before said publication

- In consequence, content of databases and even scientific literature might not reflect the actual status of the presence and availability of genetically modified organisms

- Impact on recommendation that applicants should consult databases

\section{Transfected-cell arrays}

\author{
Michal Janitz \\ University of New South Wales, Australia
}

The presentation will cover an application of transfected-cell array technology for high-throughput RNA interference functional genomics studies. Loss-of-function studies using knockout mice and gain-of-function studies using transgenic mice are a well-established approach to study gene function in in vivo situation. Besides undisputed contribution in the field of functional genomics these techniques intrinsically require a large number of animals for the production of the gene-manipulated animals as well as for the testing of gene function in the various physiologic cell populations. This requirement raises ethical issues concerning experimentation on animals, but it is also expensive due to the high cost of animal maintenance. Therefore, there is a clear need for development and optimisation of in vitro technologies, which could contribute to reduc-

tion, if not replacement, of animals in the existing knockout and transgenic mice technology. To this end, development of a high-throughput in vitro experimental approach, the transfected-cell array (TCA), in primary mammalian cells as an alternative to generation of genetically manipulated animals will be presented. We applied the RNA interference (RNAi) technology, which allows a specific inactivation of target genes. Thus, genes functions in various cell types could be investigated. The array-based method allows for high throughput functional analysis of hundreds of genes with the minimal cell number requirements. Moreover, usage of primary cells implies that experimental results can be directly transferred into in vivo situation in animal models and man. 


\title{
Gene function analysis in cell culture
}

\author{
Hiroshi Arakawa \\ Helmholtz Center Munich, Germany
}

Chicken B cell line is becoming popular model system for targeted gene disruption because of its high homologous recombination activity. I will introduce the genetic study for the molecular mechanism of immunoglobulin gene diversification

using DT40 in our lab. Some of genetic study using transgenic or knockout animals can be replaced by cell culture, if researchers are interested in the events occurring inside of the cells.

\section{Gene knock-down in zebrafish embryo: "Fishing for the molecular basis of human disease"}

\author{
Steffen Just \\ University of Heidelberg, Germany
}

Despite intensive efforts and immense medical and economic interests, the genetic causes and pathomechanisms of human diseases are mostly unknown. Understanding the complex mechanisms underlying human diseases on the molecular and physiological level is the prerequisite for the development of novel strategies for prevention, risk prediction, early diagnosis, and curative treatment. For instance, dilated cardiomyopathy (DCM) is a major cause for heart failure and sudden cardiac death. About $30 \%$ of all patients suffering from DCM carry an inherited gene defect. To date, several mutations in more than 25 genes have been identified. However, the molecular mechanisms by which these mutations lead to the pathologic features are largely unknown. Reasoning that human disease can only be adequately understood when looking at the whole organism rather than at cultured cells, whole animal models are required for an in-depth understanding of the pathogenesis and the progression of the disease. To identify novel disease causing genes and afterwards to define the disease gene-associated signalling pathways, the zebrafish particularly the zebrafish embryo has become a helpful model organism. The possibility of combining the advantages of genetic tools such as forward genetics (e.g. positional cloning) and reverse genetics (e.g. target gene knockdown) with the methods of developmental biology and cell biology makes them especially useful in basic as well as applied research. Further studies in the zebrafish will help to unveil the molecular pathomechanisms of human disease and to develop novel treatment strategies.

\section{D tissue structures using mesenchymal stem cells}

\section{Paul Genever}

Department of Biology, University of York, UK

Human multipotent mesenchymal stromal cells or mesenchymal stem cells (MSCs) are found in adult tissues such as bone marrow and are able to differentiate into osteogenic, chondrogenic and adipogenic tissues. There is intense interest in determining how MSCs may be used in future cellbased therapies, including gene therapy and tissue engineering, and as in vitro models for fundamental research and drug discovery. The intrinsic selfrenewal and differentiation capacity of MSCs allows their use in vitro to establish three dimensional (3D) tissue-like structures, such as bone and cartilage, which mimic the in vivo environment. We have developed simplified methods for cultivating hu- man MSCs under non-adherent conditions to promote cell-cell interactions to form microtissue-like structures. We have also engineered bi-differentiated osteo-chondrogenic spheroids with defined bone and cartilage features and used different biomimetic scaffolds to support osteogenic MSCs. Our 2D and 3D models of MSC growth have been used to determine pathways that regulate osteochondral differentiation using in vitro conditions that may reflect more accurately in vivo intercellular connectivity. In light of this, we have developed approaches for targeting gene disruption to allow the analysis of the effects of gene knockdown/knockout in tissue-like environments. 


\title{
D. melanogaster in toxicity screening
}

Stephan Schilling

Probiodrug AG, Halle, Germany

Pyroglutamate (pGlu)-modified $A ß$ peptides are highly abundant in sporadic and inherited Alzheimer's Disease. Formation of pGlu at the N-Terminus confers resistance against cleavage by most aminopeptidases and neprilysin, increases the cytotoxicity of the peptides and speeds up $A ß$ aggregate formation, apparently caused by a lower solubility of the modified peptides. The stabilizing effect of the pGlu-formation in case of amyloid peptides is in line with previous findings showing that glutaminyl cyclization at the N-terminus of several peptide hormones is crucial for the in vivo half life and physiological function. Our in vitro and in vivo studies provide strong evidence for a slow Glutaminyl cyclase (QC, EC 2.3.2.5) catalyzed cyclization of $\mathrm{N}$-terminal glutamic acid, substantiating a crucial role for generation of $\mathrm{pGlu}-\mathrm{A} ß$ peptides.
Several animal studies were performed for a proof of principle of QC-mediated pGlu-Aß formation. Among these, we developed a Drosophila model for generation of pGlu-Ab. The model was used to assess the efficacy of an inhibitor and the effect of inhibition on the amyloid accumulation process in these flies. Furthermore, the toxicity of the compound has been investigated using the transgenic flies. The use of the model might have implications for similar studies, which require a proof of efficacy of drug candidates to modulate biochemical or biophysical processes and to efficiently validate results obtained from other animal studies. The advantages and disadvantages of using Drosophila flies are discussed.

\section{Yeast as a model to investigate human mitochondrial diseases}

\author{
Raeka Aiyar \\ Gene Expression Unit, EMBL, Heidelberg, Germany
}

Mitochondria participate in a variety of fundamental cellular processes, including energy production, metabolism, and apoptosis. Defects in mitochondrial function can therefore be widespread and severe. The characterization of this organelle has benefited greatly from integration of functional genomics data derived from various model organisms, particularly yeast. This is due to the high conservation between the yeast and human mitochondrial systems as well as the ease of genetic manipulation and cultivation in yeast. Research in yeast has enabled the identification of mitochondrial disease genes, mechanistic analysis of disease models, and testing of disease allele phenotypes. A holistic understanding of mitochondrial disease mechanisms and how they vary across individuals, however, will require the use of transgenic animal disease models. Nonetheless, yeast serve as a good platform for a preliminary understanding of how mitochondrial function is carried out, which aspects of it are impaired in particular diseases, and predicting either genetic or chemical methods for rescuing these defects.

\section{D models of skin cancer}

\author{
Mike Philpott
}

Centre for Cutaneous Research, Barts and The London School of Medicine and Dentistry, Queen Mary College, University of London, United Kingdom

Mouse skin carcinogenesis has been described as a paradigmatic model for multistep carcinogenesis and because of ease of access this has resulted in murine models being widely used in skin cancer biology. However, malignant melanoma (MM), basal cell carcinoma (BCC) and squamous cell carcinoma (SCC) all lend themselves to in vitro $3 \mathrm{D}$ models. These models are typically based upon co-culture of keratinocytes from the epidermis on dermal scaffolds seeded with fibroblasts. Usually the dermal scaffold is composed of either de-epidermalised dermis (DED) or collagen gels. Initially used to engineer normal skin equivalents these composite cultures allow investigation of the interaction between keratinocytes and the dermis in a stratified epidermis. However, increasingly these models are being used to incorporate either cancer cells or keratinocytes retrovirally transduced with cancer genes to investigate many aspects of tumour biology including cell-cell interactions, cell proliferation and invasion. In this talk I will discuss the basic models and give examples of how they have been used in understanding biology of MM, BCC and SCC. 


\title{
Transgenic tissue cells in screening for endocrine disrupter activity
}

\author{
Gilbert Schönfelder
}

Institute of Pharmacology and Toxicology, Julius-Maximilians Universität Würzburg, Germany

With the introduction of new chemicals regulation REACH in the EU on 1 July 2007, there are not only new requirements for assessing the toxicity of new chemicals but also of approx. 30,000 existing substances. A large portion of these examined chemicals and pesticides are probably so-called endocrine-active substances (EDCs) and the question is raised whether or not EDCs may have an adverse effect on the endocrine system. These adverse effects on the environment as well as on human health are possibly mediated by (anti)estrogenic, (anti)androgenic or (anti)thyroid activities. Consequentially, to detect potential adverse effects on the endocrine and reproductive/developmental systems for patients or customers safety testing animal use has still the highest impact. By following the guidelines for regulatory safety testing, a tremendous number of animals might be required and, therefore, advanced in vitro testing methods have to be developed to reduce significantly the number of animals. In general, steroid hormones (e.g. estrogens and androgens) trigger a variety of effects mediated by slow genomic nuclear receptor mediated as well as by rapid nongenomic mechanisms. In particular, within the past decades it became accepted to test EDCs for estrogenic activity by screening for their genomic effects in vitro. Here, the transactivation activity of estrogens/ xenoestrogens regulated genes is measured with a reporter gene method, because it has been shown for some EDCs that they elicited their effects by transcriptional activation. Specifically, cells are transfected with plasmids carrying a promoter with the

\section{Transgenic hepatocytes}

\author{
Marco Tripodi \\ Università degli Studi di Roma "La Sapienza", Rome, Italy
}

Primary hepatocytes, one of the most widely used cell types for toxicological studies, have a very limited life span and must be freshly derived from mice or even humans. Attempts to use stable cell lines maintaining the enzymatic pattern of liver cells have been so far unsatisfactory. Stress proteins (heat shock proteins, HSPs) have been proposed as general markers of cellular injury and their use for environmental monitoring has been suggested. We previously described transgenic mice (Hsp70/hGH) secreting high levels of human Growth Hormone (hGH) following exposure to toxic compounds in vivo and in vitro in primary cultures derived from different organs. In addition, we also reported another transgenic model (AT/cytoMet) allowing the reproducible immortalization of untransformed hepatocytes retaining in vitro complex liver functions. From double transgenic so called estrogen responsive element (ERE) conjugated with the reporter enzyme luciferase. It is suggested, that treatment of the transfected cells with EDCs should result in an increased luciferase expression. But, in the last years several publications advanced our understanding about the pathophysiological consequences of exposure to estrogens/xenoestrogens, i.e. contradictory results were reported on the transcriptional regulation of candidate genes involved in tumor development, when cells were exposed to classical estrogens. EREs where present in their promoter sequence, but exposure of estrogen sensitive epithelial cells lead to decreased transcriptional activity and advanced, i.e. tumor growth. Therefore, the new findings have seriously to be taken into account when results from reportergene screening assays after EDCs exposure are discussed and raise several important questions: (1) Are current reportergene assays good enough for EDC screening, (2) do we have to define new physiological and pathophysiological endpoints, which might be more relevant for assessing the toxicity of EDCs, because these will make the decision on the candidate genes we have to use for creating in vitro reporter gene screening assays, (3) which cell line is appropriate to perform reportergene assays for EDC screening, (4) what do we expect from those in vitro screening assays, and (5) can we reduce animal tests by in vitro assays? In conclusion, further research has to be initiated to develop and validate new in vitro screening assays for testing the toxicity of EDCs and identifying new endocrine active chemicals. animals we derived several stable hepatic cell lines (MMH-GH) which showed a highly-differentiated phenotype as judged from the retention of epithelial cell polarity and the profile of gene expression, including hepatocyte-enriched transcription factors and detoxifying enzymes. In these cell lines, stresses induced by exposure to inorganic [Sodium Arsenite $\left(\mathrm{NaAsO}_{2}\right)$ and Cadmium Chloride $\left(\mathrm{CdCl}_{2}\right)$ ], and organic [Benzo(a)Pyrene (BaP), PentaChloroPhenol (PCP), TetraChloroHydroQuinone (TCHQ), 1-Chloro-2,4-DiNitro-Benzene (CDNB)] compounds, specifically induced hGH release in the culture medium. MMH-GH, an innovative model to evaluate the toxic potential of chemical and physical xenobiotics, provides a simple biological system that may reduce the need for animal experimentation and/or continuously deriving fresh hepatocytes. 


\title{
Genetic manipulation of rodent immune systems: prospects and problems for immunotoxicity assessment
}

\author{
Nirmala Bhogal \\ FRAME, Nottingham, United Kingdom
}

The biopharmaceutical market has grown steadily since the early 1980's and today over 150 protein biopharmaceuticals have been approved for clinical use. These products often exhibit forms of immunotoxicity that often only come to light during clinical studies. The predictive value of animal studies and traditional in vitro screens is questionable, with few existing methods able to predict immunotoxicity in a way that is useful for estimating risk for entire patient populations for a specific, and often unique, product. One of the major problems is the inherent immunogenicity of biopharmaceuticals. Genetically altered animals are beginning to feature in the expression of fully human proteins. Furthermore, a number of additional models have been developed to assess immunogenicity-related effects on biopharmaceutical efficacy, toxicity and biodistribution. The merits of these models are considered with reference to the preclinical assessment of marketed and failed biopharmaceuticals in terms of the regulatory framework, the Three Rs and current technology.

\section{Recombineering - a breakthrough technology for DNA engineering}

\author{
Youming Zhang
}

Gene Bridges GmbH, R\&D, Saarbrücken; TU Dresden, BioZ, Dresden, Germany

DNA cloning and mutation are two of the most central tasks in all Bioscience fields. Conventional DNA engineering tools like restriction and ligation have been successfully used for several decades. However, scientists still face difficulties in engineering and amplifying DNA molecules larger than $20 \mathrm{~kb}$, and therefore confront a major time-limiting factor for large DNA molecule modifications and analyses. Recently a breakthrough technique for DNA manipulation, called Recombineering or Red/ET recombination, was developed. Recombineering is mediated through homologous recombination which allows the exchange of genetic information between two DNA molecules in a precise, specific and faithful manner, qualities that are optimal for DNA engineering regardless of size. The beauty of Recombineering technology is that it only utilizes short homology sequence $(\sim 40 \mathrm{bp})$ for homologous recombination. This short homology sequences can be easily integrated into synthetic oligo primers for PCR reaction. This novel procedure allows the cloning and subcloning of almost all DNA molecules regardless of their size and composition. Recombineering is having a profound impact in the field of functional proteomics, genomics and transgeneOmics and offers a true alternative to existing techniques. It allows any DNA modification to be performed, including the insertion of point mutations, selectable and non-selectable genes, as well as the deletion of protein domains for functional analyses. All of this is done in a fraction of the time, and thus ultimately cost, that conventional methods would take. It constitutes therefore the method of choice for all DNA engineering exercises.

1. Adequate cloning technologies like for example the Red/ET recombineering technologies should be used for the cloning of transgenic constructs and for DNA constructs used for homologous recombination, allowing optimizing transgene expression and to optimally reach the aims associated with homologous recombination.

2. In some cases adequate recombineering technology like the Red/ET system will allow for the generation of genetically modified strains with better characteristics as compared to strains generated by conventional cloning technologies.

3 . Adequate cloning technologies represent a refinement measure optimising the chances of success to obtain genetically modified strains with expected properties. 


\section{ES cells based on $\mathrm{C57BL/6N}$}

\section{Johannes Wilbertz}

Department of Cell and Molecular Biology, Karolinska Center for Transgene Technologies, Karolinska Institutet, Stockholm, Sweden

The use of embryonic stem cell (ESC) lines from the inbred strain $\mathrm{C} 57 \mathrm{BL} / 6$ as a tool for gene targeting has become increasingly utilized since the availability of such cell lines. The main advantage is the possibility of avoiding a mixed 129/B6 genetic background, which is traditionally obtained in standard gene targeting experiments using 129-derived ESC lines.

The following table shows some advantages and disadvantages for the common 129 based ES-Cell lines and the C57BL/6 based ES-cell lines

\begin{tabular}{|c|c|c|}
\hline & Advantages & Disadvantages \\
\hline 129 ES-Cells & $\begin{array}{l}\text { - Easy to have access to genomic libraries } \\
\text { - Hugh variety of ES cells available } \\
\text { - Good electroporation efficiency } \\
\text { - Availability of baseline data } \\
\text { - Efficient injection } \\
\text { - Easy to get germline }\end{array}$ & $\begin{array}{l}\text { - Slower germline transmission } \\
\text { - Difficult to get coisogenic mice (129bad breeder) } \\
\text { - Long breeding process to get congenic mice (>1-3 yrs) } \\
\text { - Hugh production of "unnecessary" mice }\end{array}$ \\
\hline C57BL/6 ES & $\begin{array}{l}\text { - Fast germline transmission } \\
\text { - Easy to produce coisogenic mice } \\
\text { - Reduction of breeding / animals used ( } 1 / 5 \text { to } 1 / 10) \\
\text { - Speeds up research }\end{array}$ & $\begin{array}{l}\text { - Not so many ES cells available Cells } \\
\text { - Difficult to produce B6 ES-Cells } \\
\text { - Availability and performance of albino mice } \\
\text { - Quite often low chimeras } \\
\text { - Variation among C57BL/6 substrains }\end{array}$ \\
\hline
\end{tabular}

Even there are some disadvantages in using C57BL/6 ES-Cells it is advantageous to use B6 ES-Cells because of:

- Higher fertility / better breeding performance of C57BL/6 mice

- Direct production of coisogenic mice

- Reduction of animal numbers

- Reduction of time

- Reduction of costs
1. It is recommended that genetic modifications should directly be introduced onto well characterized inbred strains.

2. If the C57BL/6 background is compatible to the scientific approach, the use of -C57BL/6 derived embryonic stem cells (ESC) is mandatory because such cells allow for direct production of C57BL/6 co-isogenic mice.

3. With these ESC it will be possible to reduce the number of animals since no backcrossing to the C57BL/6 strain is needed. Further, time and costs are reduced.

\section{Reducing the use of GM mice by applying the Speed Backcrossing Technique}

\section{Emmanuel Gomas}

Charles River Laboratories, Saint Germain Sur l'Arbresle, France

The phenotype of GM mice is influenced by three parameters: the mutation, the housing environment and the genetic background. Thus, good management of the genetic background of a particular model is an important point to define. In particular, this could allow reducing the number of GM mice to produce and to use during phenotypic characterization of the mutation. To reach this goal, the backcross technique allows to introgress the mutation on an inbred genetic background to obtain a congenic strain.

Ten breeding generations (2.5 years) are required for a classical backcross strategy to obtain a congenic strain. But, by using a technique based on genome-wide analysis of genetic polymorphism, call speed backcross; it could be possible to reduce the number of generations ( 5 generations only) by selecting breeders with the highest rate of the preferred genetic background. But, to be sure that this technique allows gain in animal numbers, some guidelines need to be defined in the following items:

- Choice of the recipient strain
- Management of the first generation breeding scheme to fixe the genetic background of $\mathrm{X}$ and $\mathrm{Y}$ chromosomes

- Number of breeders analysed

- Number of markers analyzed

1. The use of mutant mice insufficiently backcrossed to an inbred strain for experimentalpurposes has to be considered critically.

2. The backcrossing of genetic modifications to other genetic backgrounds should be performed with as few generations as possible. Speed congenic technology allows the fast transfer of genetic modifications and should be practiced even if the number of animals used is comparable to that of standard backcrossing procedures.

3. Adequate marker density and adequate animal numbers (for selection against donor strain contamination) should be used during the speed congenic process. 


\section{Cryopreservation of transgenic mice and the 3Rs}

Johannes Schenkel

German Cancer Research Centre and Department of Physiology and Pathophysiology, Heidelberg University, Germany

The enormous scientific potential of transgenic mice, their rapidly increasing number, small populations, and the continued danger of loss make it necessary to keep these mutant lines in stock, even if they are out of current use. This housing is resource and animal consuming and needs, together with the frequent interchange of transgenic mice between different facilities, alternatives. Cryopreservation of early embryonic stages or of spermatozoa is a valuable tool to meet this challenge.

Cryopreservation of pre-implantation embryos is the most common technique. This technique is complex and the yield of embryos delivered by the donors can vary due to several reasons. These yields represent one of the most limiting factors when cryopreserving embryos. The revitalization procedure of frozen embryos is simple and in most cases successful. The assessment of the sample quality is very important, time-consuming, expensive, and will lead to a loss of samples. Microscopy, staining, over-night culture, and qualitative embryo transfer can serve for quality-assessment. In our hands, control thawing of more than 11,000 embryos out of 290 transgenic mouse lines showed a revitalization rate of about $84 \%$ with only a few variations.

Alternatively spermatozoa can be cryopreserved, too. This technique has several advantages as the enormous amount of spermatozoa/donor, the small number of donors required, the possibility to evaluate the quality using one of several parallel samples, and no need to pre-treat the donor mice. On the other hand a time and animal consuming in-vitro fertilisation (IVF) is required to recover these lines. This technique is not available for all genetic backgrounds and some of the common procedures to generate transgenic mice might negatively influence spermatozoa.

Important parameters to evaluate the quality are viability, density and motility. The state-of-art technique to assess the quality of spermatozoa is IVF; we developed animal saving control techniques and define at present the standards required.
The availability of a detailed database of cryopreserved mice (and of active mutant animal lines in a breeding nucleus) is a major help to omit redundant generation, characterization, import, or backcrosses of transgenic anmimals. Before transferring a (transgenic) mouse line to other investigators possible legal restrictions must be determined and might be solved by a material transfer agreement (MTA).

Cryopreservation of transgenic mouse lines per se omits the need to keep these lines in a breeding stockand saves enormous numbers of animals. In general, cryopreservation is strongly recommended, even if this technique is not available in-house and support of other resources is needed. Shipments of living animals can be circumvented and the import of a (transgenic) mouse line is much easier. In addition, strategies to improve embryo production and the important quality assessments can contribute to save animals, too. The availability of detailed databases helps to avoid possibly redundant work with mutant animals. Subsequently, cryopresevation and its adjacent techniques and databases are a major contribution to the 3R-principles.

1. It is highly recommended to cryopreserve genetically modified mouse strains as embryos or spermatozoa.

2 . The freezing procedure should be chosen with respect to the genetic background, the number of genetic modifications and their genotype, as well as the availability and breeding performance of the respective animals.

3. Optimized crypopreservation techniques should be used and a detailed record keeping (database) is mandatory. If such techniques are not available in house, support can be obtained by international resources as EMMA or by private companies.

4. It is recommended to ship strains only as cryopreserved samples.

5. Cryopreservation strategies are suitable for reducing the number of breeding nuclei that would otherwise be necessary to maintain the strain constantly. 


\title{
Alternatives to vasectomy and tail biopsies
}

\author{
Dirk Korthaus
}

Institute of Laboratory Animal Science and Research Center Biomodels, University of Veterinary Medicine Vienna, Austria

Glyceraldehyde 3-phosphate dehydrogenase-S, a sperm-specific glycolytic enzyme, is required for sperm motility and male fertility. Sterile Gapds-/- males in a CD1 background are even more efficient in generating plug positive foster mothers than vasectomized CD1 males. The present study was conducted to investigate several parameters that might have an effect on genotyping results, to be more confident in the use of hair follicles for DNA isolation. Tested parameters are age of mice (hair cycle stages), sampling method as well as storage of the biopsies.

Moreover, the risk of cross contamination was proved by DNA-microsatellite genome scanning. For DNA preparation different protocols were used to meet the requirements for DNA purification either for detecting a knockout allele by standard PCR or a quantitative transgene integration by realtime PCR.
However, the genotyping of 60 transgenic mouse colonies in a one year time period was not efficient and reliable using hair samples in daily work, but routinely used for second samples, as the mice are older and in a distinct hair cycle stage. Therefore, the amount of isolated DNA is much higher and the results are reliable.

1. Genetically infertile males exhibiting a good plugging performance like e.g. Gapds-/- mice may represent an alternative to vasectomized males. However, the value of such models remains to be elucidated.

2. Routine genotyping of juvenile mice using hair follicle samples is not efficient and reliable, and still is a suitable tool to re-genotype adult mice (e.g. C57BL/6 > 50 days).

\section{Humane endpoints}

\section{Kurt Bürki}

University of Zurich, Institute of Laboratory Animal Science, Switzerland

The definition of humane endpoints is an ethical requirement in modern animal experimentation: In order to avoid unnecessary suffering, animals in painful experiments have to be euthanized as soon as a predefined degree of suffering has been reached independent of whether the goal of the experiment has been reached or not. Whereas this practice is followed during the experiment, the huge numbers of animals in breeding colonies are usually not subject to predefined termination criteria. In particular for animals developing diseases due to genetic modifications the definition of early endpoints has therefore also to be defined and implemented in breeding colonies. The degree of suffering is usually classified according to a scoring system: Experimentspecific indicators of pain and stress are listed and the degree of the manifestation of each indicator is given a score. A total score is then predefined to be the tolerable limit, thus setting the endpoint. The development of a generally accepted scoring system for the animal room has to take into account that it can efficiently be applied to large number of animals by the animal caretakers, preferably during changing the cages. It has to include a limited number of easy-to-observe relevant parameters, such as overt phenotypic traits, nest structure or vocalization. The relevance of these parameters as indicators for pain and stress has however to be strengthened by further scientific investigations.

1. It is recommended to define early humane endpoints not only in experimental situations but also in breeding facilities.

2. Initially a simple, reliable and generally accepted scoring system/score sheet to detect lines with reduced welfare in the animal room has to be developed.

3. It is highly recommended to educate and train the animal technician sufficiently to be able to handle this initial scoring system.

4. To develop such an initial scoring system, research into the correlation between pain/stress and phenotypic indicators has to be strengthened.

5. Phenotypic indicators like clinical signs, behavioural changes, vocalization in the ultrasound range should be investigated to assess the possibility for implementing such indicators in the initial score system.

6. For lines with reduced welfare according to the initial scoring a specific scoring system has to be developed. In the specific scoring system the maximal acceptable total score, setting the humane endpoints, has to be defined. 


\section{Defection of pain and stress in transgenic lab animals}

\section{Paolo Cinelli}

University of Zurich, Institute of Laboratory Animal Science, Switzerland

1. Definite criteria for pain assessment are necessary for successful treatment of pain.

2. Animal caretakers and researches have to observe the animals and to identify with the help of score sheets changes in the behaviour of animals exposed to a potentially painful procedure.

3. If a person involved in the observation of the animals recognizes pain indicators more sophisticated methods like telemetry and stress hormone measurement should be applied. With the help of these technologies a pain therapy should be developed.

4. To eliminate uncertainty due to the observer's subjective judgments, rapid, reliable, and quantifiable pain detection systems have to be developed, being as less invasive as possible, and allowing an objective classification of the degree of pain.
5. Pain assessment is mandatory for pre-estimating the necessity of pain therapy in commonly used biomedical methods and to prove the efficacy of various analgesic regimes used on mice. Furthermore, the objective assessment of pain/stress in transgenic animals plays a crucial role for deciding which breeding strategy to choose in order to substantially reduce the number of affected mutant animals in breeding colonies.

6. Assessment of pain in rodents is very difficult because it is almost impossible to measure pain/stress without indirectly stressing the animals through handling.

7. Quantitative methods such as telemetry and stress hormone measurement are currently considered optimal.

8. Methods like behavioral analysis, vocalization and gene expression analysis are not yet sufficiently developed for use as a reliable diagnostic tool. Therefore, development of these technologies has to be supported.

\begin{tabular}{|c|c|c|c|c|c|}
\hline & Telemetry & Stress Hormones & Vocalization & Behavior & Gene Expression \\
\hline Rapid & - & ++ & ++ & ++ & ++ \\
\hline Objective & ++++ & ++++ & +++ & + & ++++ \\
\hline Reliable & ++++ & ++++ & $?$ & $?$ & $?$ \\
\hline Quantifiable & yes & yes & yes? & yes? & yes \\
\hline Invasive & yes & yes/no* & no & no & no* \\
\hline Animal Manipulation & no & yes/no* & yes & no & no* \\
\hline Problems & Expensive (transmitter) & Expensive (kits) & interpretation & interpretation & to early \\
\hline Individual Housing & yes & no & yes & yes/no? & no \\
\hline
\end{tabular}

